\title{
Routine bone marrow examination in the management of acute lymphoblastic leukaemia of childhood
}

\author{
CATHERINE HAWORTH, AD HEPPLESTON, PH MORRIS JONES, \\ RHA CAMPBELL, DIK EVANS, MK PALMER*
}

From the Royal Manchester Children's Hospital, Pendlebury, Manchester M27 1HA, and the *Department of Statistics, Christie Hospital and Holt Radium Institute, Withington, Manchester M20 9BX

SUMMARY Eighty-four children with acute lymphoblastic leukaemia (ALL) who had relapsed in bone marrow were studied to assess whether treatment would be more successful if relapse was detected before the disease became clinically evident. Patients whose relapse was detected by routine bone marrow examination before the disease became apparent were compared with those whose relapse was suspected from clinical examination or peripheral blood findings. In the former there was a lower percentage of blast cells in the marrow $(p<0.02)$ and the patients suffered less from complications of the disease, but there was no difference in the incidence or duration of second remissions between the two groups.

It is often assumed that early detection of relapse in leukaemia makes reinduction of remission easier. In order to detect relapse before clinically evident disease develops, many schedules, for example Medical Research Council protocols, include regular bone marrow examinations. Few children like having bone marrow aspirations performed, and these increase the work load in the clinic and laboratory. In an attempt to assess their value, we compared children whose relapse was detected only by routine bone marrow aspiration with those whose relapse was suspected from clinical examination or from examination of the peripheral blood.

\section{Patients and methods}

The status of all children treated for acute lymphoblastic leukaemia at the Royal Manchester Children's Hospital from January 1970 to June 1979 was noted. Patients who had a relapse in the bone marrow after first remission were qualified to enter into the study. Concurrent relapse in the testis or the central nervous system, however, excluded children from entry. In patients who fulfilled the above criteria the following were noted:

(1) Whether relapse was an unsuspected finding on a routine bone marrow aspirate or was found on bone marrow aspiration performed because

Accepted for publication 6 October 1980 of clinical suspicion or peripheral blood changes.

(2) The percentage of blast cells in the bone marrow when the relapse was diagnosed.

(3) Whether or not a second remission was induced.

(4) The duration of the second remission.

(5) The development of serious complications during the induction of a second remission. (A serious complication was defined as one requiring admission to hospital.)

(6) The total white cell count at initial diagnosis.

Various conventional chemotherapy regimens were used in the reinduction attempts over the nineyear period. The patients were divided into two groups. Group I were those patients in whom relapse was an unsuspected finding, while group II were patients in whom relapse was suspected from clinical examination or from examination of the peripheral blood and confirmed by bone marrow aspiration. Patients who had findings indicating relapse on a day when they were due to have a routine bone marrow examination were placed in group II.

The incidence and duration of second remissions were compared between the two groups by calculating remission duration curves using a life-table method. ${ }^{1}$ These were compared using the logrank test. ${ }^{2}$ The number and nature of serious complications in the two groups were also noted. The patients in the study were classified according to the percen- 
tage of blast cells in the bone marrow at the time of relapse and further remission curves plotted.

\section{Results}

There were 84 patients who qualified for entry into the study. In 58 patients relapse was a surprise finding on routine bone marrow examination (group I), while in 26 patients relapse was suspected on clinical grounds or from examination of the peripheral blood (group II).

Initial total white cell counts over $20 \times 10^{9} / 1$ $\left(20000 / \mathrm{mm}^{3}\right)$ were equally distributed between the two groups (20\% in group I and $18 \%$ in group II). The incidence of second remissions in the two groups was similar: 38 out of 58 patients in group I (66\%) and 16 out of 26 patients in group II $(62 \%)$. This difference was not statistically significant. The durations of second remission were also comparable (Fig. 1).

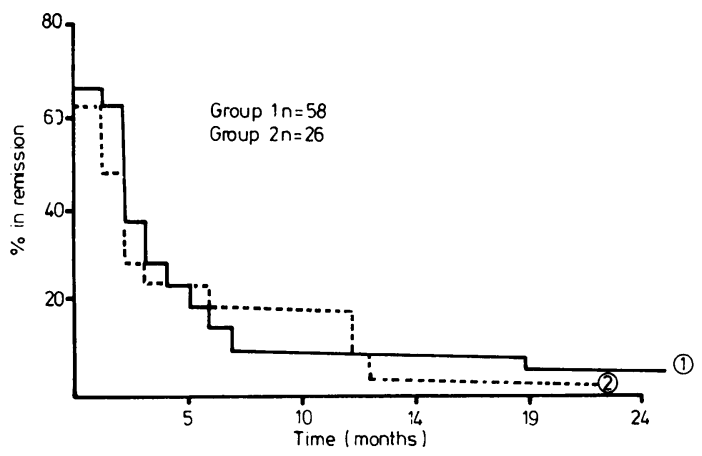

Fig. 1 Duration of remission of group I $(n=58)$ and group II $(n=26)$ patients. (The difference is not statistically significant, logrank test, $p=0 \cdot 52$.)

Group I patients tended to have fewer blast cells in the first relapse bone marrow than patients in group II (Table 1). The means were $48 \%$ (SD \pm 29$)$ (group I) and 66\% (SD \pm 31 ) (group II) and this difference was statistically significant, $p<0.02$. The

Table 1 Frequency of marrow blast cells in relapsed patients

\begin{tabular}{lll}
\hline Marrow blast cells (\%) & \multicolumn{2}{l}{ Patients (\%) } \\
\cline { 2 - 3 } & Group I & Group II \\
& $n=58$ & $n=26$ \\
\hline$<25$ & 27 & 23 \\
$25-49$ & 29 & 0 \\
$50-74$ & 17 & 15 \\
$>75$ & 27 & 62 \\
\hline
\end{tabular}

84 patients were divided into four categories accord- $\stackrel{\vec{\sigma}}{\overrightarrow{0}}$ ing to the percentage of blast cells: $(1)<25 \%$; (2) $25-49 \%$; (3) $50-74 \%$; (4) $75 \%$. No statistically significant difference (logrank test for trend, $p=0.14$ ) was found between the categories in the incidence or $\frac{\bar{D}}{\bar{N}}$ duration of second remissions (Fig. 2). When only $\frac{\bar{\sigma}}{\vec{D}}$ two categories were formed, above and below $50 \% \stackrel{\Phi}{\circ}$ blast cells, this difference was still not statistically significant $(\mathrm{p}=0 \cdot 12)$.

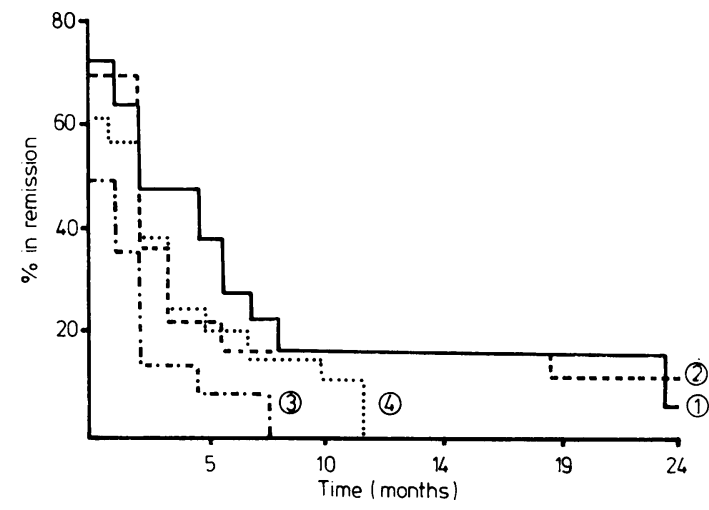

Fig. 2 Duration of remission in patients divided according to percentage of blast cells in relapse marrow. (1) $<25 \%(n=22)$; (2) $25-49 \%(n=16)$; (3) $50-74 \%$ $(n=14) ;(4)>75 \%(n=32)$. (The trend is not statistically significant, logrank test, $p=0 \cdot 14$.)

We also noted the incidence of serious complications during reinduction for the patients who? achieved a second remission. These occurred in six $\frac{5}{3}$ out of 38 patients in group I $(16 \%)$ and in seven out of the 16 patients in group II $(44 \%)$ (Table 2$)$. This difference is statistically significant $\left(\chi^{2}=4 \cdot 8, \mathrm{df}=1\right.$, $\mathrm{p}<0.05$ ). In group II patients, six of the seven $\frac{\text { ㅇ }}{2}$ complications were already present when relapse $\rightarrow$ was diagnosed and could not therefore be attributed to increased chemotherapy.

Table 2 Serious complications encountered in remission induction

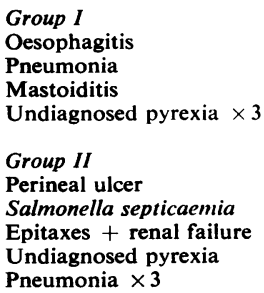




\section{Discussion}

It is generally assumed that effective cancer treatment is easier in patients with smaller malignant cell masses. There is convincing evidence for this in multiple myeloma ${ }^{3}$ and Burkitt's lymphoma. ${ }^{4}$ In acute leukaemia the position is unclear. There are reports of longer durations of remission in patients with lower blast cell counts ${ }^{5}$ whereas others ${ }^{6}$ showed no statistically significant differences in remission rate or duration in relation to peripheral blast cell count at presentation. The degree of infiltration of bone marrow at diagnosis has also been shown not to influence the length of survival ${ }^{7}$ but there is no information on leukaemia cell mass at relapse in relation to successful chemotherapy. Our results question the precept that early detection of relapse in acute lymphatic leukaemia of childhood facilitates reinduction of remission or increases the duration of second remissions.

The number of patients in the study is small, especially in group II, and we need to confirm our findings in the future with a randomised trial. The above results may be interpreted as showing that the difference in leukaemia cell mass between symptomatic and presymptomatic patients is not great enough to affect the outcome, and that within the range of preclinical to early clinically detectable disease, sensitivity to current antileukaemic drugs may be more important.
Our results suggest that routine bone marrow investigation for detecting early relapse in acute lymphatic leukaemia of childhood may be unnecessary.

\section{References}

${ }^{1}$ Kaplan EL, Meier PJ. Non-parametric estimation from incomplete observations. J Am Statist Assoc 1958;53: 457-81.

${ }^{2}$ Peto R, Pike MC, Armitage O, et al. Design and analysis of randomised clinical trials requiring prolonged observation of each patient: II analysis and examples. $B r J$ Cancer 1977;35:1-39.

${ }^{3}$ Ducie BGM, Salmon SE. A clinical staging system for multiple myeloma-correlation of measured myeloma cell mass with presenting clinical features; response to treatment and survival. Cancer 1975;36:842-54.

${ }^{4}$ Magrath I, Young JR, Anderson T, et al. Prognostic factors in Burkitt's lymphoma-importance of total tumour burden. Cancer 1980;45:1507-18.

${ }^{5}$ Keating MJ, Smith TL, Gehan EA, et al. Factors related to length of complete remission in adult acute leukaemia. Cancer 1980;45:2017-29.

${ }^{6}$ Crowther D, Beard MEJ, Bateman CJT, et al. Factors influencing prognosis in adults with acute myeloid leukaemia. Br J Cancer 1975;32:456-64.

${ }^{7}$ Gehan EA, Smith TL, Freireich EJ, et al. Prognostic factors in acute leukaemia. Semin Oncol 1976;3:271-82.

Requests for reprints to: Dr C Haworth, Department of Haematology, Royal Manchester Children's Hospital, Pendlebury, Manchester M27 1HA, England. 